Original Research Paper

\title{
A Kit for Multiplexed Detection of Antibodies to Agents of Hemotransmissible Infections
}

\author{
Alexander Poltavchenko, Anna Ersh, Pavel Filatov, \\ Oleg Nechitaylo, Yulia Krupnitzkaya and Vadim Gureev \\ Laboratory of Immunochemical Methods in Diagnostics, \\ State Research Center of Virology and Biotechnology "Vector", Koltsovo, Russia
}

\section{Article history}

Received: 10-07-2016

Revised: 03-09-2016

Accepted: 03-09-2016

Corresponding Author: Vadim Gureev

Laboratory of Immunochemical Methods in Diagnostics, State Research Center of Virology and Biotechnology "Vector", Koltsovo, Russia

Email: gureyev@ngs.ru

\begin{abstract}
The paper presents the results of laboratory trials of method using complex initial testing of antibodies to six agents of hemotransmissible infections: HIV, hepatitis $\mathrm{B}$ and $\mathrm{C}$ viruses, cytomegalovirus, Treponema pallidum and Toxoplasma gondii. The method is based on multiplexed dot immunoassay using plane protein arrays (immunochips) with the use of colloidal gold conjugates and silver development. We describe composition of the kit enabling us to conduct dot assay at room temperature within $70 \mathrm{~min}$. It was shown that the results of multiplexed analysis correlate well with data obtained using commercially available monospecific kits for ELISA. Furthermore, multiplexed dot assay is faster and more efficient in execution as compared to ELISA and can be carried out in field conditions.
\end{abstract}

Keywords: Antibodies, Multiplexed Dot Assay, Hemotransmissible Infections, Complex Initial Test, ELISA

\section{Introduction}

Among broad range of agents of infectious diseases transmitted with blood hepatitis B (HBV) and hepatitis C (HCV) viruses, Human Immunodeficiency Virus (HIV), as well as syphilis agent $(T$. pallidum) are the most prominent (Schreiber et al., 1996). Cytomegalovirus (CMV) (Volkova et al., 2015) and toxoplasmosis agent (T. gondii) are also of importance with their reproduction connected with cell blood elements. Hemotransmissible infections are widely spread among population and are able to lead to dire consequences. Course of these diseases has often the form of mixed infections in different combinations and lacks specific symptoms (Zhiburt, 2002).

Rapid and efficient detection of hemotransmissible infections is one of the important stages to control them including mandatory medical health screening of donors and groups of population with increased risk of contamination. Risk groups include healthcare professionals, patients needing longitudinal care and those who are imprisoned, family members of chronic carriers of hemotransmissible infection agents, injection drug users, homosexuals, etc. The monitoring is carried out by means of screening or diagnostic testing. Differences among those processes are caused by tasks for analyses conduction, as well as subsequent stages with their techniques being similar to each other (WHO, 2009).

Method of Immunoenzyme Assay (ELISA) is widely used in serodiagnosis of hemotransmissible infections (WHO, 2009; Zhiburt et al., 2010). However, kits for ELISA are designed to detect antibodies only to one pathogen; therefore, complex screening requires considerable time expenditures and money spending. Furthermore, some medical facilities lack necessary equipment and highly-qualified staff to carry out ELISA. Consequently, such analyses are conducted primarily in diagnostic laboratories and fast delivery of samples from remote locations to those laboratories is often a significant challenge.

Multiplexed immunodiagnostics is a new trend suggesting usage of so-called protein arrays that makes it possible to detect simultaneously multiple different analytes in investigated sample. Those arrays are often referred to as protein chips or immunochips. The main advantage of protein arrays includes the ability to study the presence of markers to broad range of infectious diseases within one analysis of clinical sample. Application of this technique can provide possibility to enhance significantly the efficiency of diagnostic methods and to reduce dramatically the cost price of analyses. 
The technology and protein arrays are considerably more complicated as compared to DNA-biochips due to a large biochemical, structural and conformational diversity among proteins, as well as a wide range of concentration of proteins in actual samples (Cretich et al., 2006; Sauer et al., 2012; Sinclair, 2013). Consequently, this diagnostic approach includes a number of technical challenges that have no optimal solutions so far. Hence, construction of each multiplexed kit requires thorough experimental studies and selection of the most effective solutions.

Previously we reported on the development of methodology for complex detection of antibodies to TORCH diseases (Poltavchenko and Yakovchenko, 2007) and evaluation of postvaccinal immunity to childhood diseases (Ersh et al., 2015) using multiplexed dot immunoassay based on plane protein arrays. This analysis is faster and cheaper compared to ELISA and makes it possible to detect concurrently a range of antibodies. This paper describes the results of laboratory testing of experimental sample of the kit for initial complex testing to detect the presence of antibodies to hemotransmissible infection agents in blood sera samples.

\section{Materials and Methods}

The following materials were used to carry out the study: Sodium azide, casein, goat monoclonal antibodies to $\gamma$-chain of human $\mathrm{IgG}$, peptone, saccharose and Tween-20 (Sigma, United States); chemical reagents of analytical purity grade; human IgG (Imtek, Russia). During the production of substrate for immunochips, we used synthetic paper based on polyvinyl chloride Pentaptint PR-M480/09-07/8101-2D8 (ForDa, Russia) (Poltavchenko et al., 2016b).

The following capture reagents was used in the analysis: Recombinant antigen HBcore of $\mathrm{HBV}$; recombinant mosaic antigen $\mathrm{NC} 34 \mathrm{ab}$ of $\mathrm{HCV}$ and recombinant antigen p150 of CMV (DiaProfMed, Ukraine); recombinant chimeric antigen gp41+gp120+gp36 of human HIV and recombinant chimeric antigen T. gondii (Fapon Biotech Inc., China); recombinant antigens $\mathrm{p} 17$ and TmpA T. pallidum (Bioservice BTC Ltd., Russia).

Working panel (ImDi, Russia) comprising 20 blood sera with or without markers of hemotransmissible infections in different combinations and concentrations was used in the work.

Experimental sample of the kit for multiplexed dot immunoassay of antibodies to hemotransmissible infection agents is constructed to conduct 20 multiplexed analyses. It includes 4 blocks with protein arrays, 4 analytical bathes, a flask with liquid component of developing system and a CD with computer program for results registration. Figure $1 \mathrm{~A}$ depicts the main components of the kit.
Substrates for protein arrays were chiseled out using typographical press in the form of blocks (combs) each comprising 5 arrays; washed by distilled water and dried out. Antigens and human immunoglobulins (positive control- $\mathrm{K}^{+}$) diluted in $0.005 \mathrm{M}$ borax buffer ( $\mathrm{pH} 8.0$ ) to $10-20 \mu \mathrm{g} \mathrm{mL}^{-1}$ were applied on each matrix as separate stains of $2.5 \mathrm{~mm}$ in diameter according to scheme in Fig. 1B. Application of $1.5 \mu \mathrm{L}$ aliquots was carried out using automatic device Dispenser-15. Blocks were dried for $12 \mathrm{~h}$ at $50^{\circ} \mathrm{C}$, blocked by immersion into $0.2 \%$ casein solution on $0.01 \mathrm{M}$ phosphate buffer ( $\mathrm{pH} 7.4)$ for $1 \mathrm{~h}$, then were dimmed into stabilizer aqueous solution (10\% saccharose, $2 \%$ peptone and $0.1 \%$ sodium azide) for 1 min, thoroughly dried, packed into polyethylene bags and stored at $4^{\circ} \mathrm{C}$.

Analytical bath is made from polypropylene using pressure molding, constructed for carrying out 5 analyses and includes 12 rows with 5 cells in each one. The bath is filled with working solutions excluding cells of the 9th row each containing $3.5 \mathrm{~mL}$ tablet of dry component of physical developer (mix of metol and citric acid by a ratio of $2: 5$ ). Cells are filled with the following solutions: Rows 2, 3, 5 and 6 contain washing solution PBS-T $(0.02 \mathrm{M}$ PBS with $0.8 \% \mathrm{NaCl}, 0.1 \%$ Tween- 20 and $0.1 \%$ sodium azide, $\mathrm{pH} 7.2$ ); row 1 contains solution for sample dilution (PBS-T with $0.02 \%$ casein, $\mathrm{pH} 8.0$ ); row 4 contains working dilution of colloidal gold in PBS-T $(20 \mathrm{~nm})$ associated with monoclonal antibodies to human $\mathrm{IgG}$; rows $7,8,10$ and 12 contain double-distilled water; row 11 contains color stabilizer, i.e., $1 \%$ thiourea in $1 \%$ dilution $\mathrm{NaOH}$ based on distilled water. Filled baths were encapsulated with Colflex material (Al Pak, Slovenia) using heat press machine CZ-PM-PL25L (China). Baths were stored at $4^{\circ} \mathrm{C}$ before usage. Supplement for the kit includes a flask with liquid component of physical developer, i.e., $0.4 \%$ dilution of silver nitrate in double-distilled water.

When applying the kit, we carry out dot immunoassay based on plane protein arrays using colloidal gold conjugates, enhancement of optical signal by silver development and its stabilization by thiourea alkaline solution. Principal scheme of dot immunoassay can be seen from Fig. 2. Dot immunoassay was carried out at room temperature in analytical baths with volume of working solutions in cells of $0.3 \mathrm{~mL}$. Before the analysis, we opened up the foil by perforator and added in the 1 st row of cells in the bath $15 \mu \mathrm{L}$ of studied blood samples and in the 8th row $150 \mu \mathrm{L}$ of double-distilled water (to dilute dry component of developer). Immunochips were immersed into the 1st row of cells and incubated for $25 \mathrm{~min}$; then they were sequentially moved to the following rows with specific time intervals: Rows 2 , 3,5 and 6 for 2 min; rows 7, 8, 10, 11 and 12 for 1 
min; row 5 for $20 \mathrm{~min}$ and row 9 for $7 \mathrm{~min}$. Just before adding immunochips into the 9 th row cells, we added there $150 \mu \mathrm{L}$ of liquid component of physical developer. After removing immunochips from the last cell, they were dried in the air and the results were registered visually according to the presence or absence of dark stains in the places of application of respective antigens. Then immunochips image was digitized using flatbed scanner and analyzed using computer program. This program enables us to detect optical density in each zone of antigen application and to percent it in relative values (i.e., percent of range from positive control-human $\operatorname{IgG}(\mathrm{K}+=100 \%)$, to negative control, i.e., area free of antigens $(\mathrm{K}-=0 \%)$ ). Besides, this program enables us to specify cutout values, i.e., critical optical density previously detected using the panel of negative sera and to print out the study protocol. Statistical processing (mean and 95\% confidence interval) and correlation analysis were carried out using Microsoft Excel.
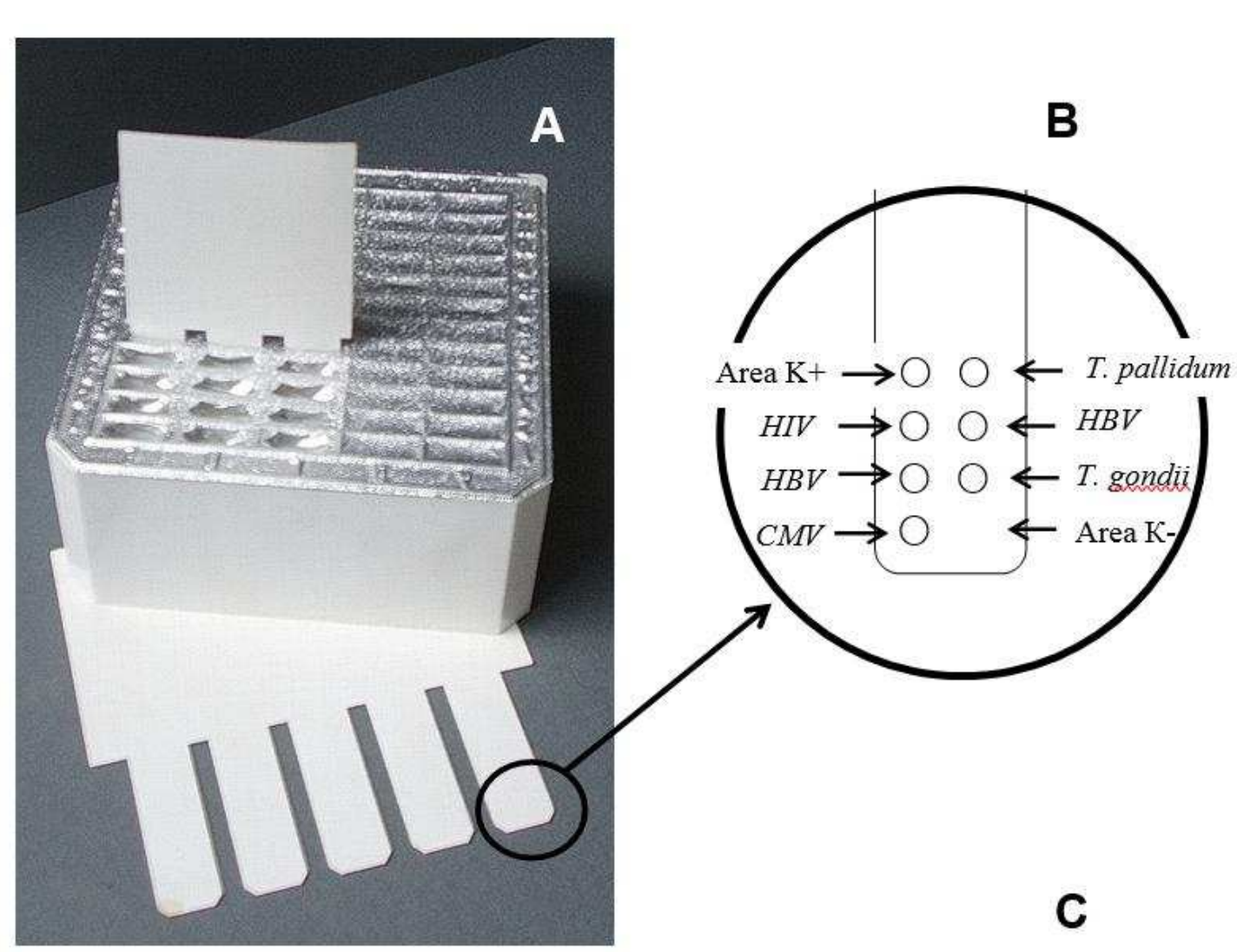

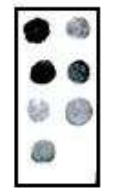

1

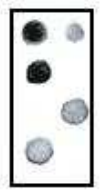

13

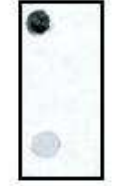

2

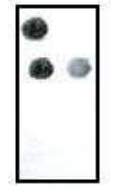

14

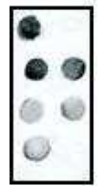

3

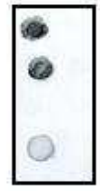

15

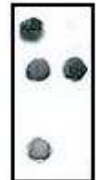

4

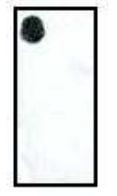

16

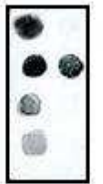

5

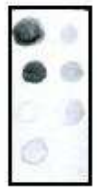

17

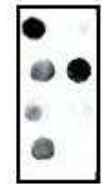

6

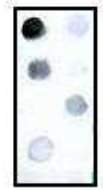

18

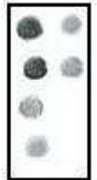

7

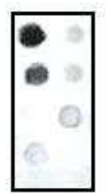

19

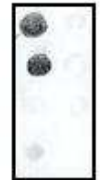

8

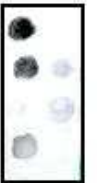

20

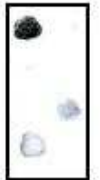

9

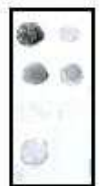

21

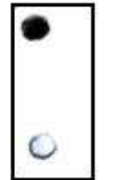

10

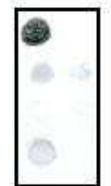

22

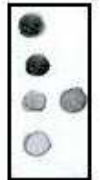

11

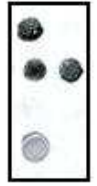

23
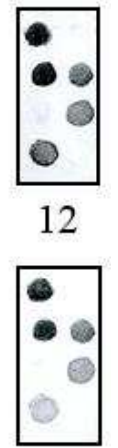

24

Fig. 1. The main elements of the kit for complex initial testing of blood products to detect the presence of antibodies to hemotransmissible infections agents (A). Scheme of application of capture reagents on immunochip (B). Results of multiplexed dot immunoassay of the samples of working panel (C). Numerals under immunochips denote numbers of panel samples 


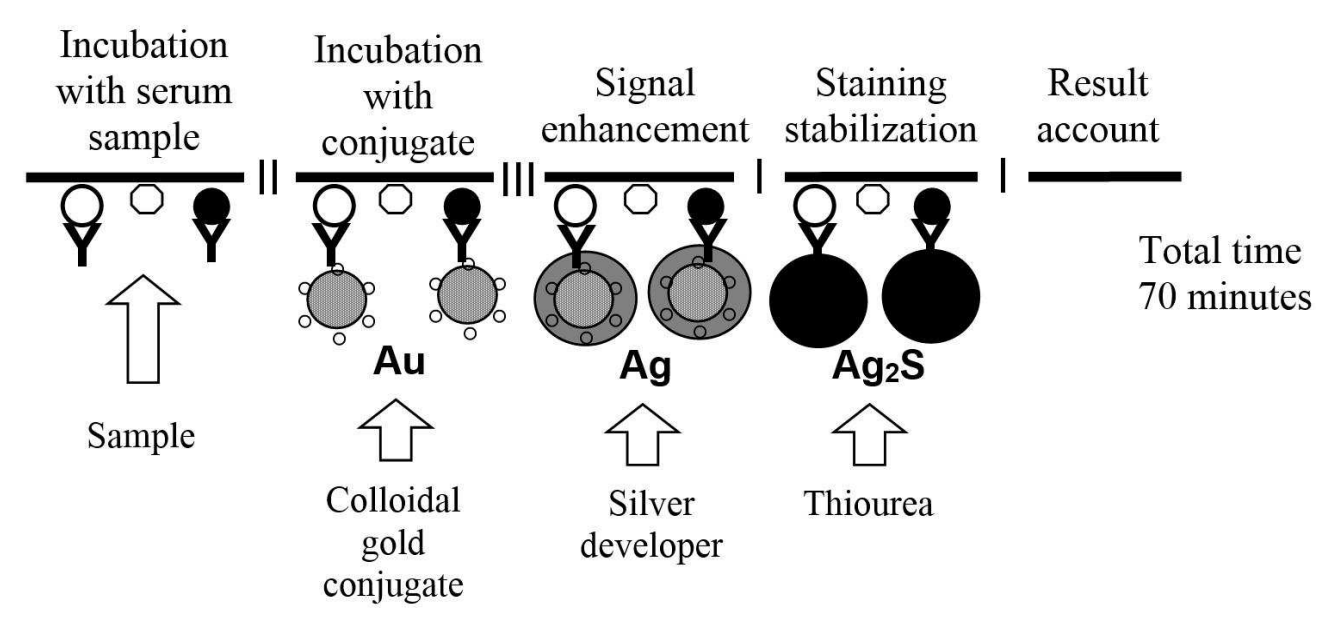

Fig. 2. Principal scheme of multiplexed dot immunoassay of antibodies using colloidal gold conjugate, enhanced signal of physical developer, and stabilized staining with alkaline solution of thiourea. I, II, III-frequency of washing

The following kits for ELISA were used as comparative tests when assessing indices of multiplexed analysis: CombiBest antiHIV-1+2, BEST antiHCV, Gepa Best gG antiHBc-IgG, RecombiBest antipalladium-IgG, VectoCMV-IgG-strip, VectoToxo-IgG (Vector-Best, Russia); Invitrologic HIV-1,2-AT, Invitrologic HBcoreAg-antibodies, HCV-DSM, Sif IgG-DS-strip, Invitrologic CMV-IgG, Melisa Toxo-IgG (Medical Biological Union, Russia). ELISA was carried out according to manufacturer's instructions. The results were registered using scanning spectrophotometer Multiscan 310 (Titerteck, Finland) at $\lambda=450 \mathrm{~nm}$.

\section{Results}

Comparison of multiplexed dot immunoassay results and data obtained using kits for ELISA designed by two commercial producers, Vector-Best (Russia) and Medical Biological Union (Russia), was implemented using working sera panel kindly furnished by ImDi (Russia). Table 1 demonstrates the results of comparative study. Protein arrays after analysis of panel samples were depicted in Fig. 1C.

Table 2 demonstrates data of correlation analysis of optical signals obtained when analyzing panel using kits for ELISA and multiplexed dot immunoassay.

\section{Discussion}

The goal of this study includes construction of a kit enabling us to rapidly conduct complex initial testing at a cheap price. Our kit makes it possible to concurrently detect 6 markers of hemotransmissible infections with three of them are subject to compulsory testing in donor blood preparations. The format of immunochip for antibody analysis fails to concurrently detect antigens so far. Therefore, we targeted antibodies to its nuclear antigen (antiHBc) for HBV instead of the 4th directive marker (HBV surface antigen). These antibodies occur in blood of infected person rather rapidly and can be regarded as circulating serologic marker of this infection (WHO, 2009).

Our system fails to differentiate antibodies in active phase of infection and postvaccinal response or previous disease. Notwithstanding, it is designed for initial testing aimed at establishing the fact of patient's exposure to infectious disease agent. If necessary, after the assay one should study the consequences of such contact in details using other diagnostic kits and/or more sophisticated confirmatory tests (Zhiburt, 2010). Practically, significant part of samples shown to be positive in initial testing fails to be verified in confirmatory analyses (Liauzheva et al., 2014) suggesting that the results obtained in different diagnostic systems are variable. We compared results of multiplexed dot immunoassay and data obtained using two kits for ELISA. Data in Table 1 shows that used kits correctly classified panel samples as positive and negative regarding HIV, HCV and HBV markers. However, when evaluating the presence of antibodies to syphilis agent Vector-Best kit failed to detect two (\#19 and \#21) positive samples. As for Medical Biological Union kit, it failed to detect antibodies to CMV in positive sera $\# \mathbf{8}$ and antibodies to $T$. gondii in 2 positive samples \#9 and 19 .

It seems that observed inconsistencies in results can be explained by the different capture and detection immune reagents used by various producers, as well as by techniques when constructing kits. In case of the usage of complicated antigenic compositions (e.g., when diagnosing syphilis) misleading results can be caused by distortion of optimal ratio of sorption mix components 
(Poltavchenko et al., 2007). Of note, different interpretation of qualitative results in discussed kits is observed while analyzing sera low in detected antibodies. Considering such inconsistencies, it is important to prevent false-negative results, since false-positive ones can only lead to extra money expenditures for confirmatory screening, while falsenegative ones can result in severe medical and social consequences. Our kit accurately detects all positive (100\% sensitivity to all markers) and negative (100\% specificity to all markers) samples and therefore has an obvious advantage over the other test systems used in the analysis.

Results obtained using these kits significantly differed in terms of value of optical signals in similar blood samples, too. Table 2 shows data of correlation analysis of optical signals obtained when analyzing panel samples by means of kits for ELISA and multiplexed dot immunoassay.

Data concerning correlation of optical signals in dot immunoassay and each system for ELISA in Table 2 are equivalent to correlation of data in those tests for ELISA. Optical signals of positive sera in immunochips look like discernable with the naked eye dark stains in the sites of application of respective antigens (Fig. 1C). Hence, registration of results of such samples can be easily implemented visually with high-level reliability.

To compare sensitivity of dot immunoassay and ELISA we prepared series of two-fold dilutions using a sample \#1 of working panel and studied dilutions by

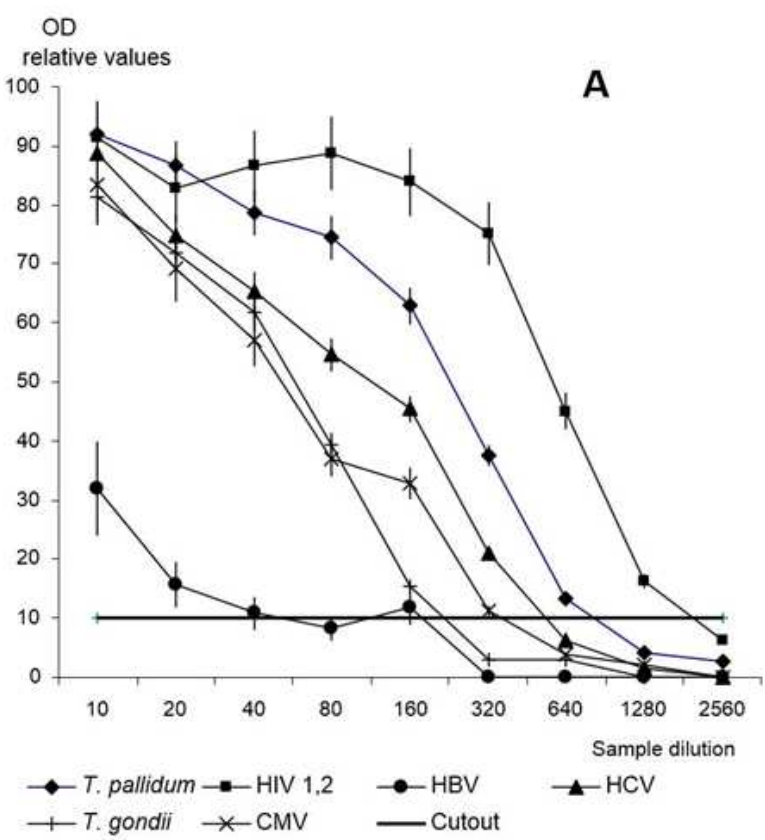

the use of kit of immunochips (or our kit) and test kit for ELISA by Vector-Best. Figure 3 depicts the results of that study demonstrating that sensitivity of dot immunoassay based on the protein arrays is equal to ELISA sensitivity. In our recent study we revealed that dot immunoassay using gold conjugates and silver enhancement of the signal provides the limit of detection of $10 \mathrm{pg}$ antibodies in a stain with dynamic range from $5 \mathrm{ng}$ to $10 \mathrm{pg}$ corresponding to a range of IgG concentration from $2.5 \mu \mathrm{L} / \mathrm{mL}$ to $5 \mathrm{ng} / \mathrm{mL}$ (Poltavchenko et al., 2016a).

Discussed data provided evidence of feasibility of efficient usage of multiplexed kit for complex initial testing of blood samples of donors and people from risk groups. Results obtained using our kit are insufficient for treatment order or implementation of administrative measures; however, our findings enable us to rapidly narrow the range of possible infections requiring further study. Multiplexed test-system can serve as a tool for both large-scale screening and individual diagnostic testing. The kit requires no additional equipment and power supply making it possible to carry out analysis in the context of delivering urgent medical aid, in remote communities lacking equipped laboratories when surgical operation or blood transmission should be conducted immediately with no possibility to deliver patient or his/her blood samples to well-equipped medical facility. We are aware of no direct analogues of described kit. The method of multiplexed analysis, composition of the kit and its separate components were reported in 5 Russian patents.

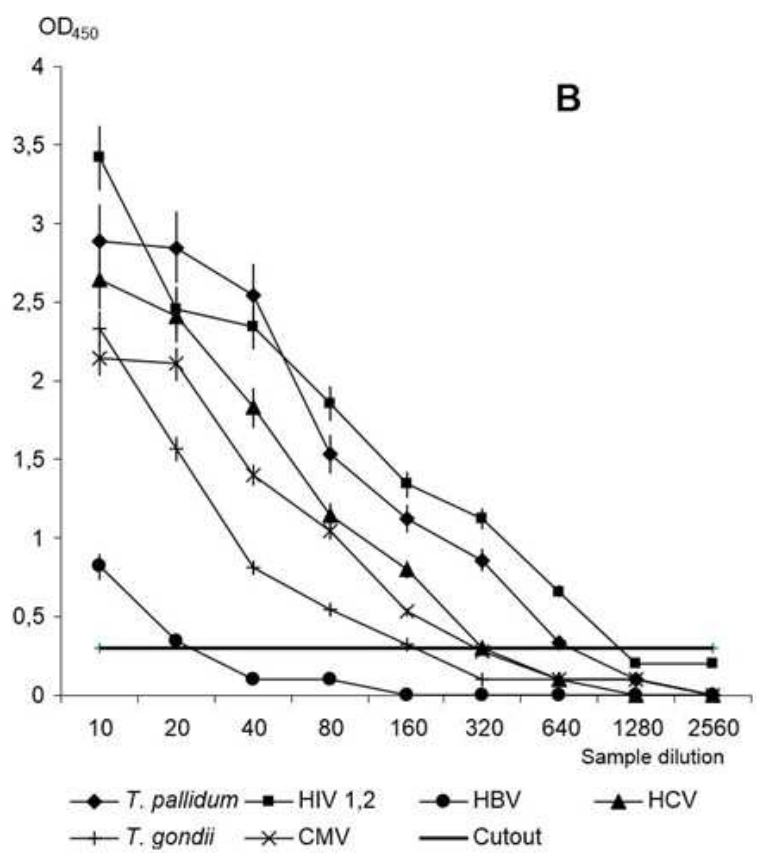

Fig. 3. Comparative evaluation of detection sensitivity of hemotransmissible diseases markers in sample \#1 of working panel using dot immunoassay based on protein arrays (A) and ELISA kits made by Vector-Best (B) 
Table 1. Results of comparative analysis of sera panel samples using kits for ELISA designed by Vector-Best (V-Best) and Medical Biological Union (MBU) and the kit for multiplexed dot immunoassay (immunochip), $n=3, p=0.95$

\begin{tabular}{|c|c|c|c|c|c|c|c|c|c|c|}
\hline \multirow{3}{*}{\multicolumn{2}{|c|}{ Agent Kit }} & \multirow{2}{*}{\multicolumn{2}{|c|}{$\begin{array}{l}\text { HIV } 1.2 \\
\text { ELISA }\left(\mathrm{OD}_{450}\right)\end{array}$}} & \multirow[b]{3}{*}{ Immunochip } & \multirow{2}{*}{\multicolumn{2}{|c|}{$\begin{array}{l}\text { Hepatitis C virus } \\
\text { ELISA }\left(\mathrm{OD}_{450}\right)\end{array}$}} & \multirow[b]{3}{*}{ Immunochip } & \multirow{2}{*}{\multicolumn{2}{|c|}{ 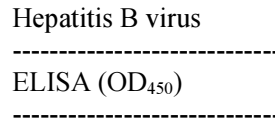 }} & \multirow[b]{3}{*}{ Immunochip } \\
\hline & & & & & & & & & & \\
\hline & & V-Best & MBU & & V-Best & MBU & & V-Best & MBU* & \\
\hline & 1 & $3.42 \pm 1.2$ & $3.16 \pm 0.6$ & $77.4 \pm 5.2$ & $2.64 \pm 1.0$ & $2.96 \pm 0.5$ & $80.8 \pm 9.7$ & $0.82 \pm 0.2$ & $0.10 \pm 0.1$ & $55.9 \pm 6.4$ \\
\hline Number & 2 & $0.17 \pm 0.1$ & $0.04 \pm 0.1$ & $0.7 \pm 1.0$ & $0.05 \pm 0.1$ & $0.04 \pm 0.1$ & $3.2 \pm 2.6$ & $0.08 \pm 0.1$ & $2.16 \pm 0.4$ & $1.3 \pm 1.0$ \\
\hline of & 3 & $3.45 \pm 0.6$ & $2.87 \pm 0.4$ & $84.0 \pm 9.3$ & $2.96 \pm 0.5$ & $3.15 \pm 0.4$ & $83.4 \pm 8.7$ & $2.14 \pm 0.4$ & $0.10 \pm 0.1$ & $52.6 \pm 8.2$ \\
\hline working & 4 & $3.41 \pm 0.3$ & $2.94 \pm 0.4$ & $85.7 \pm 5.1$ & $2.48 \pm 0.8$ & $2.55 \pm 0.8$ & $71.5 \pm 9.3$ & $2.42 \pm 0.4$ & $0.08 \pm 0.1$ & $45.4 \pm 5.6$ \\
\hline sera & 5 & $3.39 \pm 0.4$ & $3.15 \pm 0.5$ & $84.2 \pm 4.2$ & $2.54 \pm 0.4$ & $2.84 \pm 0.5$ & $77.1 \pm 6.4$ & $0.15 \pm 0.1$ & $1.99 \pm 0.3$ & $1.3 \pm 1.2$ \\
\hline \multirow[t]{19}{*}{ panel } & 6 & $3.41 \pm 1.3$ & $3.36 \pm 0.7$ & $83.8 \pm 3.6$ & $3.08 \pm 0.5$ & $3.36 \pm 0.6$ & $81.8 \pm 8.2$ & $2.84 \pm 0.3$ & $0.08 \pm 0.1$ & $45.6 \pm 4.3$ \\
\hline & 7 & $3.41 \pm 0.6$ & $3.38 \pm 0.5$ & $68.9 \pm 8.2$ & $0.45 \pm 0.2$ & $1.05 \pm 0.4$ & $53.1 \pm 6.3$ & $2.65 \pm 0.5$ & $0.08 \pm 0.1$ & $58.1 \pm 6.6$ \\
\hline & 8 & $3.43 \pm 0.8$ & $3.40 \pm 0.5$ & $60.8 \pm 6.4$ & $0.18 \pm 0.1$ & $0.04 \pm 0.1$ & $6.4 \pm 2.8$ & $0.07 \pm 0.1$ & $2.34 \pm 0.5$ & $1.9 \pm 1.3$ \\
\hline & 9 & $0.07 \pm 0.1$ & $0.10 \pm 0.1$ & $1.7 \pm 0.8$ & $0.05 \pm 0.1$ & $0.04 \pm 0.1$ & $0.7 \pm 1.0$ & $0.12 \pm 0.1$ & $2.18 \pm 0.5$ & $1.9 \pm 1.0$ \\
\hline & 10 & $0.28 \pm 0.1$ & $0.12 \pm 0.1$ & $5.2 \pm 1.4$ & $0.09 \pm 0.1$ & $0.04 \pm 0.1$ & $1.0 \pm 1.0$ & $0.12 \pm 0.1$ & $2.56 \pm 0.6$ & $0.6 \pm 1.0$ \\
\hline & 11 & $3.65 \pm 0.8$ & $3.42 \pm 0.8$ & $80.4 \pm 6.3$ & $0.05 \pm 0.1$ & $0.06 \pm 0.1$ & $2.6 \pm 1.2$ & $3.18 \pm 0.6$ & $0.45 \pm 0.5$ & $36.5 \pm 0.4$ \\
\hline & 12 & $3.65 \pm 0.7$ & $3.52 \pm 0.3$ & $84.6 \pm 5.8$ & $2.19 \pm 0.9$ & $2.49 \pm 0.5$ & $63.3 \pm 6.8$ & $0.14 \pm 0.1$ & $1.98 \pm 0.4$ & $0.8 \pm 1.0$ \\
\hline & 13 & $3.68 \pm 0.7$ & $3.53 \pm 0.6$ & $87.0 \pm 6.9$ & $0.05 \pm 0.1$ & $0.05 \pm 0.1$ & $1.9 \pm 1.6$ & $0.17 \pm 0.1$ & $1.90 \pm 0.4$ & $2.5 \pm 2.0$ \\
\hline & 14 & $3.69 \pm 0.2$ & $3.46 \pm 0.4$ & $52.2 \pm 4.2$ & $2.48 \pm 0.4$ & $2.19 \pm 0.6$ & $35.8 \pm 2.8$ & $0.06 \pm 0.1$ & $1.99 \pm 0.5$ & $4.3 \pm 2.4$ \\
\hline & 15 & $3.65 \pm 1.5$ & $3.23 \pm 0.5$ & $70.2 \pm 9.2$ & $0.06 \pm 0.1$ & $0.04 \pm 0.1$ & $1.2 \pm 1.0$ & $0.10 \pm 0.1$ & $2.52 \pm 0.4$ & $0.6 \pm 1.0$ \\
\hline & 16 & $0.08 \pm 0.1$ & $0.04 \pm 0.1$ & $1.3 \pm 1.2$ & $0.05 \pm 0.1$ & $0.05 \pm 0.1$ & $3.0 \pm 2.0$ & $0.08 \pm 0.1$ & $2.14 \pm 0.3$ & $4.3 \pm 1.6$ \\
\hline & 17 & $2.45 \pm 0.4$ & $2.14 \pm 0.3$ & $64.0 \pm 5.6$ & $2.41 \pm 0.6$ & $2.12 \pm 0.5$ & $34.5 \pm 3.4$ & $0.15 \pm 0.1$ & $2.30 \pm 0.1$ & $3.7 \pm 1.5$ \\
\hline & 18 & $2.21 \pm 0.5$ & $2.56 \pm 0.5$ & $57.9 \pm 5.3$ & $0.06 \pm 0.1$ & $0.04 \pm 0.1$ & $3.0 \pm 2.2$ & $0.09 \pm 0.1$ & $2.13 \pm 0.6$ & $1.9 \pm 1.0$ \\
\hline & 19 & $2.34 \pm 0.5$ & $2.98 \pm 0.5$ & $52.0 \pm 5.3$ & $1.83 \pm 0.4$ & $2.14 \pm 0.5$ & $16.8 \pm 4.1$ & $0.09 \pm 0.1$ & $1.41 \pm 0.4$ & $1.8 \pm 1.0$ \\
\hline & 20 & $2.36 \pm 0.6$ & $2.42 \pm 0.4$ & $48.2 \pm 3.4$ & $1.62 \pm 0.6$ & $1.63 \pm 0.3$ & $18.4 \pm 2.3$ & $0.07 \pm 0.1$ & $2.64 \pm 0.5$ & $1.3 \pm 1.2$ \\
\hline & 21 & $1.86 \pm 0.4$ & $2.12 \pm 1.1$ & $37.4 \pm 3.3$ & $1.14 \pm 0.3$ & $1.35 \pm 0.3$ & $26.7 \pm 1.7$ & $0.07 \pm 0.1$ & $1.88 \pm 0.4$ & $5.5 \pm 1.5$ \\
\hline & 22 & $1.52 \pm 0.6$ & $2.36 \pm 0.3$ & $36.1 \pm 6.6$ & $1.02 \pm 0.4$ & $0.55 \pm 0.3$ & $18.1 \pm 7.6$ & $0.07 \pm 0.1$ & $2.17 \pm 0.3$ & $5.5 \pm 1.5$ \\
\hline & 23 & $1.89 \pm 0.5$ & $2.12 \pm 0.5$ & $57.4 \pm 6.5$ & $2.68 \pm 0.4$ & $3.21 \pm 0.6$ & $58.7 \pm 5.2$ & $0.17 \pm 0.1$ & $2.71 \pm 0.6$ & $3.4 \pm 1.3$ \\
\hline & 24 & $1.99 \pm 0.6$ & $1.56 \pm 0.5$ & $49.3 \pm 9.1$ & $2.32 \pm 0.6$ & $2.61 \pm 0.6$ & $41.2 \pm 8.0$ & $0.16 \pm 0.2$ & $2.14 \pm 0.4$ & $2.9 \pm 0.4$ \\
\hline \multirow[t]{26}{*}{ Cutout } & & 0.30 & 0.24 & 10.0 & 0.25 & 0.24 & 10.0 & 0.27 & 0.80 & 10.0 \\
\hline & & \multicolumn{2}{|c|}{ Treponema pallidum } & & \multicolumn{2}{|c|}{ Cytomegalovirus } & & \multicolumn{2}{|c|}{ Toxoplasma gondii } & \\
\hline & 1 & $2.89 \pm 0.3$ & $3.12 \pm 0.5$ & $85.1 \pm 6.2$ & $2.14 \pm 0.6$ & $1.82 \pm 0.3$ & $36.6 \pm 6.4$ & $2.33 \pm 0.5$ & $2.11 \pm 0.3$ & $64.5 \pm 4.2$ \\
\hline & 2 & $0.11 \pm 0.1$ & $0.06 \pm 0.1$ & $5.1 \pm 2.4$ & $0.20 \pm 0.6$ & $0.2 \pm 0.2$ & $6.4 \pm 3.3$ & $0.08 \pm 0.1$ & $0.09 \pm 0.1$ & $0.8 \pm 0.6$ \\
\hline & 3 & $0.06 \pm 0.1$ & $0.10 \pm 0.1$ & $6.5 \pm 2.1$ & $2.54 \pm 0.5$ & $2.11 \pm 0.4$ & $68.0 \pm 4.9$ & $2.52 \pm 0.4$ & $2.47 \pm 0.6$ & $72.5 \pm 6.1$ \\
\hline & 4 & $0.05 \pm 0.1$ & $0.06 \pm 0.1$ & $4.9 \pm 2.2$ & $1.70 \pm 0.3$ & $1.65 \pm 0.4$ & $34.8 \pm 5.5$ & $0.27 \pm 0.2$ & $0.12 \pm 0.1$ & $9.2 \pm 1.0$ \\
\hline & 5 & $0.06 \pm 0.1$ & $0.06 \pm 0.1$ & $6.6 \pm 2.8$ & $2.48 \pm 0.3$ & $2.51 \pm 0.3$ & $68.2 \pm 8.3$ & $0.10 \pm 0.1$ & $0.08 \pm 0.1$ & $4.7 \pm 1.0$ \\
\hline & 6 & $0.07 \pm 0.1$ & $0.06 \pm 0.1$ & $1.0 \pm 0.6$ & $2.18 \pm 0.3$ & $2.36 \pm 0.4$ & $63.7 \pm 5.2$ & $0.09 \pm 0.1$ & $0.10 \pm 0.1$ & $1.3 \pm 1.0$ \\
\hline & 7 & $0.42 \pm 0.1$ & $1.11 \pm 0.4$ & $46.0 \pm 9.1$ & $2.24 \pm 0.4$ & $2.21 \pm 0.6$ & $51.9 \pm 5.2$ & $0.10 \pm 0.1$ & $0.10 \pm 0.1$ & $0.7 \pm 1.0$ \\
\hline & 8 & $0.06 \pm 0.1$ & $0.07 \pm 0.1$ & $0.1 \pm 0.1$ & $0.46 \pm 0.1$ & $0.15 \pm 0.1$ & $21.2 \pm 1.0$ & $0.07 \pm 0.1$ & $0.10 \pm 0.1$ & $2.1 \pm 1.0$ \\
\hline & 9 & $0.07 \pm 0.1$ & $0.08 \pm 0.1$ & $6.4 \pm 2.2$ & $1.86 \pm 0.4$ & $2.31 \pm 0.3$ & $50.4 \pm 6.8$ & $1.15 \pm 0.3$ & $0.30 \pm 0.2$ & $17.4 \pm 2.3$ \\
\hline & 10 & $0.06 \pm 0.1$ & $0.07 \pm 0.1$ & $6.2 \pm 2.0$ & $2.12 \pm 0.3$ & $2.46 \pm 0.5$ & $54.9 \pm 5.2$ & $0.08 \pm 0.1$ & $0.08 \pm 0.1$ & $1.3 \pm 0.5$ \\
\hline & 11 & $0.06 \pm 0.1$ & $0.06 \pm 0.1$ & $3.2 \pm 0.8$ & $2.46 \pm 0.4$ & $2.85 \pm 0.5$ & $61.3 \pm 5.2$ & $1.95 \pm 0.4$ & $1.05 \pm 0.2$ & $31.5 \pm 2.5$ \\
\hline & 12 & $0.06 \pm 0.1$ & $0.06 \pm 0.1$ & $2.0 \pm 1.0$ & $2.75 \pm 0.5$ & $3.12 \pm 0.4$ & $64.6 \pm 4.4$ & $2.65 \pm 0.4$ & $2.26 \pm 0.4$ & $72.0 \pm 4.6$ \\
\hline & 13 & $2.56 \pm 0.4$ & $2.32 \pm 0.5$ & $54.4 \pm 5.4$ & $2.78 \pm 0.5$ & $3.01 \pm 0.4$ & $68.4 \pm 5.8$ & $2.44 \pm 0.3$ & $2.43 \pm 0.5$ & $69.7 \pm 5.2$ \\
\hline & 14 & $0.06 \pm 0.1$ & $0.06 \pm 0.1$ & $1.3 \pm 1.0$ & $0.20 \pm 0.1$ & $0.1 \pm 0.1$ & $4.3 \pm 1.0$ & $0.08 \pm 0.1$ & $0.08 \pm 0.1$ & $0.8 \pm 1.0$ \\
\hline & 15 & $0.07 \pm 0.1$ & $0.1 \pm 0.1$ & $0.6 \pm 0.6$ & $1.53 \pm 0.4$ & $2.13 \pm 0.3$ & $43.6 \pm 8.4$ & $0.09 \pm 0.1$ & $0.09 \pm 0.1$ & $1.3 \pm 1.0$ \\
\hline & 16 & $0.05 \pm 0.1$ & $0.06 \pm 0.1$ & $1.1 \pm 0.6$ & $0.2 \pm 0.1$ & $0.12 \pm 0.1$ & $5.4 \pm 0.2$ & $0.09 \pm 0.1$ & $0.08 \pm 0.1$ & $0.8 \pm 1.0$ \\
\hline & 17 & $0.87 \pm 0.2$ & $1.95 \pm 0.4$ & $47.5 \pm 4.8$ & $2.11 \pm 0.2$ & $1.63 \pm 0.5$ & $23.4 \pm 2.6$ & $1.57 \pm 0.2$ & $1.04 \pm 0.3$ & $37.4 \pm 2.9$ \\
\hline & 18 & $0.68 \pm 0.2$ & $0.80 \pm 0.1$ & $24.2 \pm 2.6$ & $1.22 \pm 0.1$ & $1.89 \pm 0.2$ & $25.4 \pm 3.3$ & $2.14 \pm 0.6$ & $2.00 \pm 0.8$ & $49.3 \pm 3.1$ \\
\hline & 19 & $0.27 \pm 0.1$ & $0.90 \pm 0.3$ & $28.1 \pm 4.2$ & $1.40 \pm 0.5$ & $1.45 \pm 0.7$ & $23.1 \pm 3.5$ & $0.51 \pm 0.1$ & $0.2 \pm 0.1$ & $27.7 \pm 2.5$ \\
\hline & 20 & $0.05 \pm 0.1$ & $0.06 \pm 0.1$ & $1.9 \pm 1.0$ & $1.69 \pm 0.3$ & $1.95 \pm 0.5$ & $30.0 \pm 2.6$ & $0.79 \pm 0.2$ & $0.48 \pm 0.1$ & $25.5 \pm 2.0$ \\
\hline & 21 & $0.27 \pm 0.2$ & $0.92 \pm 1.1$ & $24.7 \pm 1.5$ & $2.04 \pm 0.6$ & $2.12 \pm 0.4$ & $36.4 \pm 0.8$ & $0.24 \pm 0.1$ & $0.18 \pm 0.1$ & $7.4 \pm 0.7$ \\
\hline & 22 & $0.04 \pm 0.1$ & $0.09 \pm 0.1$ & $3.0 \pm 1.1$ & $1.21 \pm 0.6$ & $0.76 \pm 0.2$ & $17.1 \pm 1.0$ & $0.06 \pm 0.1$ & $0.12 \pm 0.1$ & $5.6 \pm 0.9$ \\
\hline & 23 & $0.04 \pm 0.1$ & $0.10 \pm 0.1$ & $4.0 \pm 3.2$ & $1.09 \pm 0.2$ & $0.87 \pm 0.3$ & $16.7 \pm 1.9$ & $0.09 \pm 0.1$ & $0.09 \pm 0.1$ & $4.2 \pm 2.7$ \\
\hline & 24 & $0.04 \pm 0.1$ & $0.09 \pm 0.1$ & $1.9 \pm 0.8$ & $0.96 \pm 0.3$ & $0.98 \pm 0.2$ & $18.2 \pm 2.8$ & $2.13 \pm 0.4$ & $1.72 \pm 0.3$ & $40.2 \pm 7.2$ \\
\hline Cutout & & 0.30 & 0.25 & 10.0 & 0.30 & 0.27 & 10.0 & 0.30 & 0.30 & 12.0 \\
\hline
\end{tabular}

List of kits for ELISA is described in Materials and Methods; positive results of detection are shaded grey; distinct results are typed bold.

* Only results with values $<$ cutout are considered to be positive.

Results of dot immunoassay are given using relative values. 
Table 2. Correlation coefficients for data from Table 1 obtained using different kits for detecting G class antibodies to HIV, HCV, HBV, CMV, as well as to syphilis (T.pallidum) and toxoplasmosis (T. gondii) agents

\begin{tabular}{|c|c|c|c|c|}
\hline Agent & Kit & V-Best & MBU & Immunochip \\
\hline \multirow[t]{3}{*}{ HIV } & V-Best & 1.000 & 0.964 & 0.944 \\
\hline & MBU & 0.964 & 1.000 & 0.910 \\
\hline & Immunochip & 0.944 & 0.910 & 1.000 \\
\hline \multirow[t]{3}{*}{$\mathrm{HCV}$} & V-Best & 1.000 & 0.982 & 0.869 \\
\hline & MBU & 0.982 & 1.000 & 0.916 \\
\hline & Immunochip & 0.869 & 0.916 & 1.000 \\
\hline \multirow[t]{3}{*}{$\mathrm{HBV}$} & V-Best & 1.000 & 0.806 & 0.872 \\
\hline & MBU & 0.806 & 1.000 & 0.876 \\
\hline & Immunochip & 0.872 & 0.876 & 1.000 \\
\hline \multirow[t]{3}{*}{ T. pallidum } & V-Best & 1.000 & 0.933 & 0.892 \\
\hline & MBU & 0.933 & 1.000 & 0.981 \\
\hline & Immunochip & 0.896 & 0.981 & 1.000 \\
\hline \multirow[t]{3}{*}{ CMV } & V-Best & 1.000 & 0.935 & 0.900 \\
\hline & MBU & 0.935 & 1.000 & 0.904 \\
\hline & Immunochip & 0.900 & 0.904 & 1.000 \\
\hline \multirow[t]{3}{*}{ T. gondii } & V-Best & 1.000 & 0.965 & 0.961 \\
\hline & MBU & 0.965 & 1.000 & 0.969 \\
\hline & Immunochip & 0.961 & 0.969 & 1.000 \\
\hline
\end{tabular}

\section{Conclusion}

The kit for multiplexed dot immunoassay of antibodies to hemotransmissible disease agents can provide complex approach to diagnosis of hemotransmissible infectious diseases and dramatically simplify conduction of initial testing, to make it more rapid, cheap and available for patients.

\section{Acknowledgement}

This work was supported by the Russian Ministry of Education and Science within the framework of a Federal Targeted Program for Research and Development in Priority Areas of Development of the Russian Scientific and Technological Complex for 20142020, agreement no. 14.607.21.0020 (RFMEF160714X0020).

\section{Authors' Contributions}

All authors have read and approved the final manuscript.

Alexander Poltavchenko: Designed the study, structured and planned the paper.

Oleg Nechitaylo, Pavel Filatov, Anna Ersh, Yulia Krupnitzkaya and Vadim Gureev: Processed the data, analyzed and discussed the findings, wrote the paper.

\section{Ethics}

The paper contains unpublished material. The corresponding author confirms that there were no ethical issues concerning the manuscript.

\section{References}

Cretich, M., F. Damin, G. Pirri and M. Chiari, 2006. Protein and peptide arrays: Recent trends and new directions. Biomolecular Eng., 23: 77-88. DOI: 10.1016/j.bioeng.2006.02.001

Ersh, A.V., A.G. Poltavchenko, S.A. P'yankov, A.P. Agafonov and N.A. Krivenchuk et al., 2015. The multiplex method of estimation of humoral immunity to vaccine regulated childhood infections. Prob. Virol., 60: 41-45. PMID: 26021074

Liauzheva, F.M., R.S. Tlenkopachev and E.B. Zhiburt, 2014. Blood donor deferral due to infections in Kabardino-Balkaria. Transfuziologiya, 15: 40-46.

Poltavchenko, A.G. and A.M. Yakovchenko, 2007. Multiplexed serodiagnostics of infectious diseases. 4. Laboratory trials of the multiplexed test prototype. Biotechnol. Russia, 3: 117-124.

Poltavchenko, A.G., A.M. Yakovchenko, O.N. Nadtochiy, N.A. Krivenchuk and A.N. Rybakov, 2007. Development of a serum panel with the rated content of IgG antibodies to antigens p17 and p41 of Treponema pallidum. Vestnik Dermatologii i Venerologii, 3: 5-13.

Poltavchenko, A.G., A.V. Ersh and Y.A. Krupnitzkaya, 2016a. The selection of the detection system for multiplexed dot-immunoassay of antibodies. Russian Clin. Laboratory Diagnost., 4: 229-233.

Poltavchenko, A.G., B.N. Zaitsev, A.V. Ersh, O.S. Taranov and D.V. Korneev et al., 2016b. Selection of substrate material for protein arrays. Protect. Metals Phys. Chem. Surfaces, 52: 209-215.

Sauer, U., J. Pultar and C. Preininger, 2012. Critical role of the sample matrix in a point-of-care protein chip for sepsis. J. Immunol. Methods, 378: 44-50. DOI: 10.1016/j.jim.2012.02.002 
Schreiber, G.B., M.P. Busch, S.H. Kleinman and J.J. Korelitz, 1996. The risk of transfusiontransmitted viral infections. New England J. Med., 334: $1685-1690$.

DOI: 10.1056/NEJM199606273342601

Sinclair, J.C., 2013. Constructing arrays of proteins. Curr. Opin. Chem. Biol., 17: 946-951. DOI: 10.1016/j.cbpa.2013.10.004

Volkova, S.D., V.N. Chebotkevich, G.Y. Kir'yanova, E.I. Kaytandzhan and V.V. Burylev et al., 2015. An algorithm for providing ill persons of risk group with cytomegalovirus-negative hemocomponents. Transfuziologiya, 16: 34-39.
WHO, 2009. Screening Donated Blood for TransfusionTransmissible Infections: Recommendations. 1st Edn., World Health Organization, Geneva, ISBN-10: 924154788X, pp: 67.

Zhiburt, E.B., 2002. Transfusion Medicine. 1st Edn., Petersburg, Piter, ISBN-10: 5-94723-281-2, pp: 736.

Zhiburt, E.B., E.A. Klyuyeva, M.N. Gubanova and E.A. Shestakov, 2010. Peculiarities of national security of infectious safety of blood products. Vestnik Roszdravnadzora, 2: 64-66. 